\title{
Spinal Anesthesia Facilitates the Early Recognition of TUR Syndrome
}

\author{
Sam McGowan-Smyth Nikhil Vasdev Shan Gowrie-Mohan \\ Department of Urology and Anaesthetics, Lister hospital, Stevenage, UK
}

\section{Key Words}

Transurethral resection of the prostate $\cdot$ Complication . Anesthesia

\begin{abstract}
Objectives: To investigate what clinical features typically present in transurethral resection (TUR) syndrome and to see which classically present first. The purpose of the study was to establish whether or not a particular method of anesthesia is preferred in detecting this syndrome in its early stages. Methods: A total of 1,502 transurethral resection of the prostate (TURP) over a 15 year period were reviewed to see which, if any, went on to experience this complication. Of these cases, 48 developed TUR syndrome. The case records were reviewed retrospectively and the presenting clinical features were analysed. All TURPs were routinely performed under spinal anesthesia and followed a standardised set up. The irrigation fluid used in all operations was Glycine $1.5 \%$. Results: Forty eight patients displayed clinical features of TUR syndrome giving an incidence of 3.2\%. Trainees of varying experience caused all but one case. Median resection time, resection weight and volume of intraoperative glycine irrigation fluid were 55 minutes (range 40-75 minutes), 44 grams (range 24-65 g), and $28 \mathrm{I}$ (24-48 I) respectively. Only 16/48 TURPs had a recorded capsular perforation. Pre- vs. post-operative median hematocrit, hemoglobin and serum sodium were 0.42 vs. $0.33,14.2 \mathrm{~g} / \mathrm{dl}$ vs. $10.1 \mathrm{~g} / \mathrm{dl}$ and 142 $\mathrm{mmol} / \mathrm{l}$ vs. $121 \mathrm{mmol} / \mathrm{l}$ respectively. Patients presented with nausea 44/48, vomiting 28/48, visual disturbance 29/48,
\end{abstract}

\section{KARGER}

Fax +4161306 1234

E-Mail karger@karger.ch

www.karger.com
(C) 2015 S. Karger AG, Basel

1015-9770/14/0092-0057\$38.00/0

Accessible online at:

www.karger.com/cur apprehension 37/48, disorientation $17 / 48$, breathing difficulties $17 / 48$, and bradycardia 19/21. The earliest observed sign was nausea $21 / 48$, then bradycardia $11 / 48$, apprehension 11/48, and visual disturbance 10/48; after which the procedure was abandoned. None of the patients developed stupor, coma or seizures. Out of the 48 patients, 9 were admitted to high dependency units and all of these were treated with IV furosemide. One patient required a blood transfusion. All patients recovered within 48 hours (range 18-48 hours) and none had any long term complications on follow up. Conclusion: The features most associated with the early presentation of TUR syndrome require the patient to be conscious for detection. The use of spinal anaesthesia is therefore desirable to facilitate its early recognition.

Copyright $\odot 2015$ S. Karger AG, Basel

\section{Introduction}

Transurethral resection of the prostate (TURP) remains the surgical gold standard for the treatment of benign prostatic hyperplasia. Approximately 40,000 operations are performed annually in the UK. Whilst generally the operation is considered safe, transurethral resection (TUR) syndrome is a rare but potentially dangerous complication that is observed in $0.7-1.4 \%$ of all prostate resections $[1,2]$. TUR syndrome is a term applied to a constellation of clinical features caused primarily by 
excessive absorption of the irrigation fluid used during endoscopic procedures [3]. Absorption of this fluid is an unpredictable complication of TURPs which proves treacherous when greater volumes directly correlate to more severe symptoms and a poorer prognosis caused largely by the effects of hypoosomolarity and hyponatremia [4].

Symptoms range from nausea, vomiting, apprehension, disorientation and visual disturbances to the more severe symptoms associated with large volumes of fluid absorption including stupor, seizures, or coma. The latter are likely to require an admission to intensive care. Severe TUR syndrome is rare; however, it carries a mortality risk of up to $25 \%$ [3]. Anesthetists must be aware of these symptoms in order to promptly diagnose this syndrome. This is facilitated by the use of spinal anesthesia.

\section{A Retrospective Study}

A recent study at Lister Hospital reviewed 1,502 TURPs over a 15-year period. Of these cases, 48 developed TUR syndrome. The case records were reviewed retrospectively; the details of which will follow the subsequent discussion. All TURPs were routinely performed under spinal anaesthesia and followed a standardised set up. The irrigation fluid used in all operations was Glycine $1.5 \%$.

\section{TUR Syndrome}

TUR syndrome is a multifactorial iatrogenic syndrome that arises from the absorption of large volumes of irrigation fluid, typically during a TURP. In the UK, the most commonly used irrigant is $1.5 \%$ glycine [5]; a hypotonic solution which is used to visualise and distend the operative site and to remove debris and blood. Whilst it is commonly thought that the syndrome results solely from dilutional hyponatremia, fluid overload and the effects of glycine toxicity contribute significantly to the pathophysiology of the condition [6].

\section{Why Glycine?}

An irrigation fluid must be non-conductive (to prevent dispersion of the diathermy current), non-hemolytic (so that hemolysis does not occur should it enter the circulation), and transparent (so that the surgeon's view is not distorted). For these reasons the fluid cannot contain electrolytes but also cannot be pure water.
$1.5 \%$ Glycine is a non-electrolyte solution with an osmolarity of $220 \mathrm{mOsmol} / 1$ [5]. This makes it hypotonic with respect to plasma.

\section{Glycine Absorption}

During a TURP, glycine is absorbed directly and immediately into the peri-prostatic venous plexus. It is also absorbed more slowly through the peri-vesical and retroperitoneal spaces. Typically fluid absorption occurs at a rate of $20 \mathrm{ml} / \mathrm{min}$ so during an operation of average length, patients should be expected to absorb 1-1.5 1 of glycine. Volumes of absorption up to four times this size however, have been recorded. The average amount of absorbed hypotonic fluid is usually easily dealt with in a healthy individual, with $90 \%$ of glycine being metabolised to ammonia, glycolic acid and water by the liver, and the remaining $10 \%$ being metabolised by the kidney [6].

In clinical practice, it is almost impossible to accurately assess the amount of glycine absorbed though it can be predicted via the assessment of other factors:

Prostate size and duration of surgery-the greater duration of surgery, the greater the volume of glycine absorbed. Problems are most frequently associated with operations lasting over an hour with a prostate size of more than $50 \mathrm{~g}$.

Infusion pressure-the height of the glycine bag should be kept as low as possible to ensure adequate irrigation without excessive infusion pressures. Higher pressures increase absorption.

Blood loss-a large volume of blood loss suggests a high number of open veins.

Venous pressure-hypovolemia and hypotension increase the rate of fluid absorption [7].

Capsular perforation or bladder perforation-this allows large volumes of irrigation fluid into the peritoneal cavity where it is rapidly absorbed [4].

Operations typically take 30 to 60 minutes to complete though TUR syndrome can occur as quickly as $15 \mathrm{~min}-$ utes after resection begins [8]. It is therefore important to optimise the factors mentioned above to reduce the risk of this potentially dangerous syndrome developing.

\section{Symptoms of TUR Syndrome}

The clinical presentation of TUR syndrome can be explained through the absorption of large volumes of $1.5 \%$ glycine causing a dilutional hyponatremia, fluid overload and glycine toxicity.

Dilutional hyponatremia results in symptoms of agitation, confusion, nausea, seizure and coma. The severity of symptoms reflects the degree of hyponatremia; ce- 
rebral herniation and death can result if left untreated. Additionally, glycine induces an osmotic diuresis which results in absolute losses of sodium from the body, and this can be further exacerbated by the release of atrial natriuretic peptide which promotes natriuresis [6].

Fluid overload presents with signs of the subsequent pulmonary edema and heart failure. Patients initially develop hypertension, dyspnea, chest pain and cyanosis. Rapid absorption of large volumes of glycine can lead to reflex bradycardia and marked decrease in systolic arterial pressure.

Glycine itself is a non-essential amino acid which functions as an inhibitory neurotransmitter in the retina. Excess amounts slow down the transmission of action potentials from the retina to the cerebral cortex, with prolongation of visual evoked potentials and deterioration of vision occurring after as little as several hundred milliliters of glycine has been absorbed. If the patient is conscious under spinal anesthesia, they may report seeing flashing lights or other visual disturbances. Facial warmth and prickling sensations are also early signs of glycine absorption. When glycine concentrations are increased, bradycardia can result from its cardiotoxic effects. The features of prolonged glycine toxicity include hypotension and coma [6].

The incidence and severity of symptoms directly correlates with the volume of glycine absorbed. In one retrospective analysis [9], patients who absorbed $0-300 \mathrm{ml}$ of glycine on average developed more than one symptom. When 1-2 1 had been absorbed, this increased to more than two symptoms, and to more than three when 2-3 1 had been absorbed, reaching more than 5 symptoms for volumes over 31 . This has been corroborated in subsequent prospective studies $[8,11]$.

\section{Study Results}

In our study, 48 patients out of the 1,502 TURPs displayed clinical features of TUR syndrome giving an incidence of $3.2 \%$. Trainees of varying experience caused all but one case. Median volume of intraoperative glycine irrigation was 281 (range 24-48 1), with a median resection time and resection weight of 55 minutes (range 40-75 minutes) and $44 \mathrm{~g}$ (range 24-65 g) respectively. Pre- vs. post-operative median serum sodium concentration was $142 \mathrm{mmol} / \mathrm{l}$ vs. $121 \mathrm{mmol} / \mathrm{l}$, with a median hematocrit and hemoglobin change from 0.42 to 0.33 , and 142 to $101 \mathrm{~g} / \mathrm{l}$ respectively. Of the 48 TURPs, only 16 recorded a capsular perforation.

The patients presented with nausea 44/48, apprehension $37 / 48$, visual disturbance $29 / 48$, vomiting $28 / 48$, disorientation 17/48, dyspnoea $17 / 48$, and bradycardia
$19 / 21$. Of these, the earliest observed sign was nausea $21 / 48$, bradycardia $11 / 48$, visual disturbance $10 / 48$ and apprehension 11/48, after which the procedure was abandoned. None of the patients developed symptoms of stupor, seizures or coma associated with the most severe presentation of the syndrome. Out of the 48 patients, 9 were admitted to high dependency units and all of these were treated with IV furosemide. One patient required a blood transfusion.

All patients recovered within 48 hours (range 18-48 hours) and none had any long term complications on follow up.

The majority of the clinical features of TUR syndrome require the patient to be conscious for them to be detected. Spinal anaesthesia therefore facilitates its early diagnosis. Under general anaesthesia, the only evidence may be the late and non-specific signs of tachycardia and hypotension.

\section{Anesthesia}

Spinal anesthesia is considered to be the anesthetic of choice for TURPs, though the procedure can be completed just as successfully under a general anesthetic. Short term morbidity and mortality and long term outcomes are similar irrespective of the technique used [12]. Spinal anesthesia however, may offer several important advantages.

\section{Spinal Anesthesia}

This method confers good postoperative analgesia and helps reduce the stress response to surgery [4]. It is also particularly useful for patients with significant respiratory disease. Of greater significance, a spinal anaesthetic allows for the recognition of any changes in conscious level in addition to any symptoms that may develop. It therefore makes it the ideal choice for the early recognition of TUR syndrome. Early detection of capsular tears and bladder perforation is also possible with a spinal as the patient may complain of shoulder or periumbilical pain given the spinal level is limited to T10 [5]. A spinal block to this level is also sufficient to eliminate the discomfort caused by bladder distention.

Severe hypotension is uncommon with spinal anesthesia [4]. The lithotomy position can compensate for sympathetic block by increasing venous return, though masked hypotension can later be revealed when the legs are returned to the supine position. Any hypotension should be treated with vasoconstrictors rather than fluid to reduce the risk of fluid overload. 


\section{General Anesthesia}

Circumstances such as the patient having contraindications to spinal anesthesia, a persistent cough, or being unable to lie in the supine position for a long period of time may lead one to prefer the method of general anesthesia. Functional residual capacity and tidal volume are reduced by a combination of the lithotomy position and head-down tilt, though these can be counteracted by tracheal intubation and positive pressure ventilation [4].

Diagnosis of TUR syndrome may prove more problematic as patients will be unable to complain of the early symptoms leaving clinicians to rely on the later heart rate and blood pressure changes for detection.

\section{Local Anesthesia}

On very rare occasions, TURP in high risk patients has been performed using local infiltration of the perineum and prostatic fossa. Spinal is many times preferred as lumbar anesthesia may miss sacral segments. This method is not recommended and alternative treatments should be explored.

\section{Management}

\section{Prevention}

Any pre-existing hyponatremia should be diagnosed and treated before a TURP is undertaken. The risk factors previously mentioned should be optimised to ensure the risk of developing TUR syndrome is minimal. Efforts should be made to ensure the patient is euvolemic prior to starting. Intravesicular pressure must be kept as low as possible; the infusion bag should not be kept higher than $100 \mathrm{~cm}$ above the bladder and it is important to maintain sufficient outflow therefore one must ensure that the catheter is not kinked or blocked. Any bleeding points identified should be coagulated. The duration of surgery should try and be kept under an hour whenever possible. In prolonged procedures, it would be wise for the anesthetist to consider prophylactic furosemide to offload the excess of fluid that may have been absorbed.

\section{Detection}

As previously discussed, early signs of the syndrome developing are best detected when the patient is awake under spinal anaesthesia [4]. Several of the clinical factors described earlier, such as agitation and nausea will only become apparent if the patient is conscious. Regular monitoring of their blood pressure, heart rate, and ECG are also essential for picking up the signs of excess fluid absorption.
If TUR syndrome is suspected, an arterial or venous blood gas would provide a quick assessment of their serum sodium.

\section{Treatment}

Mild cases of detected TUR syndrome can often be treated supportively in addition to a dose of intravenous furosemide. Although this loop diuretic can worsen hyponatraemia, it results in relative loss of more water than sodium, thus decreasing fluid overload and increasing serum sodium levels [7]. Typically a dose of $40 \mathrm{mg}$ is given but more may be required given the delayed absorption of glycine through the retroperitoneal and perivesical space. In mild cases, the operation should be finished as quickly as possible and any haemorrhages should be controlled.

Rarely, severe cases can occur if early signs are not detected or if initial treatment is inadequate. In cases such as these, surgery must be abandoned as soon as possible and any intravenous fluids stopped. Treatment should involve respiratory and cardiovascular support with early involvement of the intensive therapy unit team. Bradycardia and hypotension should be treated with atropine, adrenergic drugs and intravenous calcium [4]. Any seizures require immediate intervention with intravenous anticonvulsants such as diazepam or lorazepam, with intravenous magnesium being considered if these fail.

Correction of hyponatremia should be done cautiously. Both severe acute hyponatremia and rapid correction of hyponatremia can lead to permanent neurological damage, most notably central pontine myelinolysis. Those with an acute fall in serum sodium below $120 \mathrm{mmol} / \mathrm{l}$ accompanied by neurological signs should be considered for administration of hypertonic saline $(\mathrm{NaCl} 1.8-3 \%)$ [13]. Typically, $3 \%$ saline is given at a rate of $1.2-2.4 \mathrm{ml} /$ $\mathrm{kg} / \mathrm{h}$ until there is symptomatic improvement. This will bring about a rise in serum sodium of approximately 1-2 $\mathrm{mmol} / \mathrm{l} / \mathrm{h}$, though one must be extremely vigilant not to exceed a rise of $12 \mathrm{mmol} / \mathrm{l}$ in 24 hours. Once a sodium of $>120 \mathrm{mmol} / \mathrm{l}$ is achieved, further improvements via fluid restriction and intravenous furosemide are usually sufficient.

\section{Conclusion}

The combination of dilutional hyponatraemia and fluid overload make TUR syndrome a rare but potentially dangerous consequence of TURP. The severity of symptoms is dependant on the degree of hyponatraemia and 
those that occur earliest require patient consciousness for detection. Three out of the four earliest presenting symptoms would not be detected if the patient is under general anaesthesia. Although severe cases are rare, mortality is high unless recognised and treated promptly. For this reason, spinal anaesthesia is preferred to ensure the delay anticipated with general anaesthesia is avoided. Irrespective of method, a high index of suspicion of TUR syndrome is necessary for a timely diagnosis and effective management.

\section{References}

1 Zepnick H, Steinbach F, Schuster F: Value of transurethral resection of the prostate (TURP) for treatment of symptomatic benign prostatic obstruction (BPO): an analysis of efficiency and complications in 1015 cases. Aktuelle Urol 2008;39:369-372.

2 Reich O, Gratzke C, Bachmann A, Seitz M, Schlenker B, Hermanek P, Lack N, Stief CG: Morbidity, mortality and early outcome of transurethral resection of the prostate: A prospective multicenter evaluation of 10,654 patients. J Urol 2008;180:246-249.

3 Hahn RG: Fluid absorption in endoscopic surgery. Br J Anaesth 2006;96:8-20.

4 O'Donnell A, Foo I: Anaesthesia for transurethral resection of the prostate. Contin Educ Anaesth Crit Care Pain 2009;9:92-96.
5 Lynch M, Anson K: Time to rebrand transurethral resection of the prostate? Curr Opin Urol 2006;16:20-24.

6 Arya M, Shergill IS, Kalsi JS, Muneer A, Mundy AP: Viva practice for the FRCS (Urol) examination. London, Radcliffe Publishing, 2010

7 Allman K, McIndoe A, Wilson I: Oxford Handbook of Anaesthesia, ed 3. Oxford, Oxford University Press, 2011.

8 Gravenstein D: Transurethral resection of the prostate (TURP) syndrome: a review of the pathophysiology and management. Anesth Analg 1997;84:438-446.

9 Olsson J, Nilsson A, Hahn RG: Symptoms of the transurethral resection syndrome using glycine as the irrigant. J Urol 1995; 154:123128.
10 Hahn RG, Sandfeldt L, Nyman CR: Double-blind randomized study of symptoms associated with absorption of glycine $1.5 \%$ or mannitol 3\% during transurethral resection of the prostate. J Urol 1998;160:397-401.

11 Hahn RG, Shemais H, Essén P: Glycine 1.0\% versus glycine $1.5 \%$ as irrigating fluid during transurethral resection of the prostate. $\mathrm{Br} \mathrm{J}$ Urol 1997;79:394-400.

12 Reeves MD, Myles PS: Does anaesthetic technique affect the outcome after transurethral resection of the prostate? BJU Int 1999;84:982-986.

13 Kumar S, Berl T: Sodium. Lancet 1998;352: 220-228. 